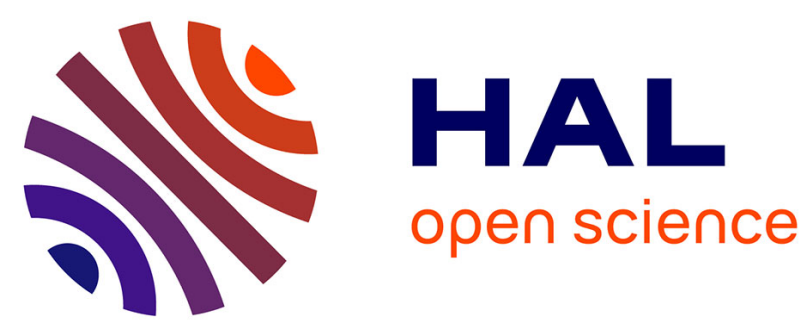

\title{
Entre Crise et Rebond: Questions autour de la durabilité des systèmes de production cotonniers au Mali
}

Isabelle Droy, Jean-Etienne Bidou, Jean-François Belieres

\section{To cite this version:}

Isabelle Droy, Jean-Etienne Bidou, Jean-François Belieres. Entre Crise et Rebond: Questions autour de la durabilité des systèmes de production cotonniers au Mali. The European Journal of Development Research, 2012, The European Journal of Development Research, 3 (24), pp.491-508. 10.1057/ejdr.2012.12 . hal-02486970

\section{HAL Id: hal-02486970 https://hal.science/hal-02486970}

Submitted on 21 Feb 2020

HAL is a multi-disciplinary open access archive for the deposit and dissemination of scientific research documents, whether they are published or not. The documents may come from teaching and research institutions in France or abroad, or from public or private research centers.
L'archive ouverte pluridisciplinaire HAL, est destinée au dépôt et à la diffusion de documents scientifiques de niveau recherche, publiés ou non, émanant des établissements d'enseignement et de recherche français ou étrangers, des laboratoires publics ou privés. 


\title{
Entre crise et rebond : questions autour de la durabilité des systèmes de production cotonniers au Mali
}

\author{
Isabelle Droy, IRD - UMI Résiliences \\ Jean Etienne Bidou, UMR ADES \\ Jean-François Bélières, CIRAD - UMR ART-Dev
}

\section{Résumé}

Le système de production articulé autour du coton et des céréales a été développé au Mali dans le cadre d'une filière intégrée, à l'amont et à l'aval, permettant de sécuriser l'environnement socio-économique. La profonde transformation du système agraire et des agricultures familiales a été considérée, sur trois décennies, comme une réussite en termes de développement. Examiné sous l'angle de la durabilité, ce modèle présentait cependant des faiblesses économiques, sociales et environnementales qui se sont exacerbées avec la baisse, depuis plusieurs années des prix de vente du coton-fibre sur le marché mondial. Mais, les réformes actuelles de la filière et les alternatives ne sont pas elles-mêmes exemptes de contradictions entre les différentes dimensions de la durabilité.

Mots-Clés : coton, pauvreté, Mali, durabilité, système agraire

\section{Abstract}

A production system based on cotton and cereals has been developed in Mali within a frame of an integrated supply chain securing the socio-economic environment. For three decades, the deep transformation of the rural structures in the Malian cotton belt has been considered as a success in terms of development. But, looked under the light of sustainable development, this model showed economic, social and ecological flaws which increased and became obvious in the recent years when the price of cotton went down on the world markets. Nevertheless, the current supply chain reforms are far from being flawless, and do not seem to provide alternatives in terms of sustainability.

Keywords: cotton, poverty, Mali, sustainability, farm system 
$J E L: O 13, Q 18, Q 56, O 18$.

\section{Introduction}

Le développement agricole durable peut être défini comme l'ensemble des processus de transformation des systèmes de production qui permettent d'accroître et d'améliorer à court terme les revenus et les conditions de vie des populations exploitant les productions agricoles, forestières et pastorales, tout en préservant la cohésion sociale et les conditions écologiques nécessaires au maintien et à la diversification future à moyen ou long terme de ces productions (Mathieu, 2001). Ainsi, comme d'autres paysans érigés en figures emblématiques du développement durable, le producteur malien de coton devrait assumer les trois missions: poursuivre la croissance économique, préserver l'environnement, et promouvoir le lien social. Or, dans un contexte de pauvreté ${ }^{1}$, de dépendance vis-à-vis des marchés extérieurs, de croissance démographique élevée et de contraintes climatiques fortes, le défi s'est révélé particulièrement lourd à relever.

Il est reconnu que le «système coton » diffusé dans le cadre d'une filière intégrée verticalement a été le moteur des transformations sociales et économiques de toute une région (Raymond, 1989, Hugon, 2005a). A ce titre il a pu être considéré comme un modèle de développement dans une Afrique où les réussites agricoles sont rares à l'échelle régionale. Ces mutations rapides ont cependant engendré des déséquilibres plus ou moins marqués selon les zones du bassin de production du cotonnier et qui font douter de la durabilité de ce modèle.

Frappé par la chute des cours mondiaux, aux prises avec des problèmes de gestion de la filière (Hugon, 2005b) et avec l'exigence de privatisation venant des institutions de Bretton Woods, le système est entré dans une crise profonde qui dure depuis le début des années 2000. Alors que les cours mondiaux du coton sont remontés en 2010 à des niveaux élevés et 
que la Banque Mondiale met moins d'intransigeance dans sa politique, le système de production cotonnier malien est-il capable de se reconstruire?

Dans la première partie, nous rappellerons quelques étapes de la construction de ce modèle basé sur le développement des agricultures familiales. Ensuite, en se plaçant au niveau de l'unité de production agricole, nous en soulignerons les limites sous l'éclairage des différentes composantes de la durabilité. Enfin, nous montrerons les difficultés de rétablissement de la filière, alors que les perturbations externes qui ont amorcé la crise se sont provisoirement éloignées.

\section{Les trente glorieuses du bassin cotonnier}

Il a fallu un demi-siècle pour construire le système cotonnier tel qu'il fonctionnait à la fin des années 1990. La région actuelle de production était, dans les années 1950, une marge peu peuplée du pays bambara; elle est devenue un foyer de population. Cultivé au sein d'exploitations familiales, le coton est devenu le pivot d'un nouveau système de production, plus intensif. Véritable «moteur» du développement économique et social dans les villages, son impact macro-économique a été très fort (ODHD, 2009) en fournissant l'essentiel des exportations agricoles du Mali durant la décennie 1990, alors que les surplus en céréales de la région cotonnière contribuaient à la sécurité alimentaire du reste du pays. Cependant, depuis 1999, les principales recettes d'exportation du pays ne proviennent plus du coton, mais de l'or, résultat des investissements réalisés dans le secteur minier et de cours mondiaux favorables.

\subsection{Le développement de la filière coton.}

Durant la période coloniale, l'échec de la culture du coton irrigué dans la zone de l'Office du Niger a conduit l'administration à développer sa culture en pluvial. La Compagnie Française de Développement des fibres Textiles (CFDT) a dès les années 1950 vulgarisé un système de culture plus intensif avec la culture pure du cotonnier, la traction animale et l'utilisation de la fumure organique. 
La production de coton n'a réellement pris son essor qu'avec la création de la Compagnie Malienne de Développement des Textiles (CMDT) en 1974 et la mise en place d'une filière intégrée et administrée (comme pour d'autres pays africains de la zone franc). La société cotonnière contrôlée par l'État avait en charge l'ensemble des activités amont et aval de la production, un monopole et une obligation d'achat, des modalités de stabilisation des prix et des relations contractuelles avec les autres acteurs en particulier les producteurs (Fok, 1999). La zone de production du coton en culture pluviale, initialement au Sud-Est du Mali, a été étendue pour occuper aujourd'hui la plus grande partie du Sud du pays ${ }^{2}$ (figure 1). La production augmente régulièrement jusqu'à la dévaluation en 1994, où elle double rapidement pour atteindre plus de 600000 tonnes en 2004 (figure 2). Ce sont alors 190000 exploitations familiales qui s'y consacrent, soit plus du quart des exploitations agricoles du pays (CPS, 2008) et 2,5 à 3 millions de personnes vivaient en partie des revenus de cette culture.

Le développement de la production cotonnière a souvent été mis à l'actif de l'organisation très intégrée de la filière avec un «système coton» assurant l'approvisionnement en intrants (engrais, pesticides), la commercialisation des graines de coton à un prix garanti annoncé en début de campagne, l'accès aux crédits, l'appui et l'encadrement des producteurs à travers des organisations villageoises et la mise en place d'infrastructures économiques. Ce mode d'organisation a fait l'objet de nombreuses analyses, et si des faiblesses ont été relevées, nul ne conteste la réussite agricole qu'il a entraînée (Raymond, 1989, Fok, 1994, Hugon, 2005a, OECD, 2006, Egg et al., 2008, Tschirley et al., 2009).

\subsection{Une évolution rapide des systèmes de production}

Le développement de la culture du cotonnier correspond à une évolution profonde des exploitations agricoles familiales du Sud du Mali, (Fok, 1994, Deveze et Halley des Fontaines, 2005). 
Le processus le plus marquant est le passage d'un système traditionnel d'agriculture d'abattis-brûlis (successions culturales entrant en rotation avec des périodes plus ou moins longues de jachère) à un système de culture permanente (Gigou et al., 2004, Dufumier et Bainville, 2006). Cette mutation est liée à la généralisation du labour en traction animale et de la fertilisation minérale et organique. La fertilisation minérale a nettement et régulièrement progressé, y compris après la dévaluation du FCFA en 1994 qui avait fortement renchéri le prix des intrants importés. La fertilisation organique a accompagné l'accroissement du cheptel bovin et la diffusion des techniques de compostage.

Les exploitations ont évolué à la fois dans leur structure et leur fonctionnement. Beaucoup ont capitalisé en augmentant leur équipement (matériels de traction animale notamment) et leur cheptel. Sur la longue période, la part des exploitations peu ou pas équipées a fortement diminué. L'accroissement de la part du coton dans les assolements est net et il s'est accompagné d'une augmentation des surfaces consacrées au maïs, plus productif que le mil ou le sorgho, et réagissant mieux à l'apport de fumure.

\subsection{La structuration du monde rural}

La structuration du monde rural a évolué avec les associations villageoises (AV). Les premières AV ont été créées au milieu des années 1970 dans un contexte de crise de confiance liée aux détournements de fonds de certains employés de la CMDT au détriment des paysans lors de la commercialisation et de la récupération du crédit (Belloncle, 1993). Leur mission principale a été d'assurer la commercialisation primaire du coton-graine, la distribution des intrants et la diffusion d'informations techniques. Elles ont aussi assumé une responsabilité de développement local par le financement d'investissements collectifs (centre d'alphabétisation, eau potable, école, centre de santé) et celui d'activités communautaires (entretien des forages, paiement des matrones ou infirmiers, etc.). Leurs ressources étaient assurées par le paiement des frais de marché et les excédents de pesée versés par la CMDT. Ces organisations, sans 
personnalité juridique établie, ont été généralisées avec le principe de « une AV par village encadré ». En raison de difficultés de gestion et d'organisation interne, beaucoup d'entre elles ont éclaté (Kébé et Sidibé-Kébé, 1998).

A la fin des années 1990, la CMDT a promu la création d'Associations de Producteurs de Coton (APC) avec un statut juridique qui les assimilait à des coopératives : en 2003, il y avait un peu moins de 7000 organisations de producteurs de coton dans toute la zone cotonnière, dont environ $20 \%$ d'APC.

La création des syndicats, au début des années 90 à la faveur de la démocratisation du pays, a été une des réponses des producteurs à leur absence de représentation dans le pilotage de la filière (Bélières et al., 2008). Les luttes engagées par les producteurs pour défendre leurs intérêts ont été un moyen de pression puissant tout au long des années 2000, avec notamment la grève des semis qui a provoqué la baisse de production de l'année 2000 (figure 2).

La privatisation de la filière a été une des mesures adoptées sous la pression des organisations internationales pour faire face à la crise cotonnière au début des années 2000 . Dans ce cadre, il était prévu d'accorder aux producteurs une part du capital des sociétés agroindustrielles et dans cette perspective, avec l'appui financier de bailleurs de fonds, les APC et les AV ont été fortement incitées à se transformer en Coopératives fédérées au sein de l’Union Nationale des Sociétés Coopératives de Producteurs de Coton (UN-SCPC). Cette restructuration a été faite sans que ne soient véritablement analysés les besoins et la demande des producteurs.

Sans se prononcer sur la situation actuelle, et même si l'on manque d'études spécifiques sur les impacts économiques et sociaux de ces organisations, de nombreux auteurs reconnaissent qu'elles ont assumé, avec efficacité, les fonctions technico-économiques de gestion des intrants, de commercialisation du coton et de relais pour la vulgarisation agricole 
et qu'elles ont été les vecteurs des principaux investissements collectifs locaux au moins jusqu'à la décentralisation avec la mise en place des Communes en 1999.

\subsection{Un choc extérieur : baisse des cours du coton sur le marché mondial}

Tout au long de son existence, la filière a traversé des crises qui ont contribué à sa structuration, mais jusqu'ici, les interventions de l'Etat et des partenaires financiers (notamment l'Agence Française de Développement) avaient permis de passer ces épisodes. La crise qui prévaut depuis le milieu des années 2000 est cependant plus grave.

Le coton-fibre étant presque totalement exporté, le Mali subit les fluctuations du marché mondial sur lequel il n'a pas de prise. Il subit également les fluctuations du taux de change entre euro et US dollar. Après un pic atteint au milieu des années 1990, les cours mondiaux du coton se sont dégradés (figure 3). En 1999, cette baisse a été répercutée en cours d'année alors que les prix avaient déjà été fixés : la CMDT, affaiblie par les malversations et erreurs de gestion des années précédentes, s'est révélée incapable de mettre en œuvre les mécanismes de soutien des prix (Hugon, 2005b). Les agriculteurs répondirent à cette rupture du contrat par une grève des semis de coton, mouvement qui fut si massif que la production chuta de moitié en 2000, entraînant un déficit financier catastrophique pour la CMDT et de graves conséquences économiques sur l'ensemble de l'économie nationale en 2001. Dès lors, le gouvernement malien, sous la pression des bailleurs de fonds, et en particulier de la Banque Mondiale, a dû engager un plan de réforme visant le démantèlement de la filière intégrée avec le désengagement de la CMDT de nombreuses activités et sa privatisation, toujours inachevée, formalisée dans la «Lettre de politique de développement de la filière coton » (MDR, 2001).

La poursuite de la baisse des cours mondiaux a aggravé la crise. En 2004, le maintien, certainement par peur des conséquences sociales, du prix d'achat au producteur fixé à 210 CFA/kg en début de campagne, a creusé les pertes de la CMDT. En 2005, un nouveau 
mécanisme de fixation du prix a été adopté prenant en compte les cours mondiaux et remettant en question le système de prix garanti en début de campagne (Nubukpo, 2011). Il s'est traduit par une baisse brutale des prix à 160 CFA/kg (figure 4). Un nouveau dispositif de soutien des prix au producteur a été mis en place en 2007, géré par 1’UN-SCPC.

Ces réformes n'ont pas enrayé la crise de la filière. La production a chuté fortement, l'approvisionnement en intrants est assuré de façon très inégale. Les organisations de producteurs sont souvent très fortement endettées et traversent des crises majeures en raison de la difficile gestion de la caution solidaire dès que de nombreux membres sont concernés par des apports de coton graine insuffisants pour faire face aux remboursements. Le paiement aux producteurs est souvent effectué avec retard. La CMDT elle-même a été segmentée en quatre filiales qui vont être privatisées. L'Etat a pris en charge la plus grande partie du passif de la société avant le découpage en quatre filiales et a mis en œuvre un plan social. Un appel d'offre international a été lancé ; le processus est toujours en cours mais peine à avancer, la filière est en attente des évolutions institutionnelles programmées. Les incertitudes sur le devenir de cette filière perdurent. Par ailleurs, la question de l'efficacité de la libéralisation des secteurs cotonniers resurgit dans divers travaux (Tschirley, 2010).

A l'échelle de la filière, la durabilité du système coton est clairement questionnée, mais c'est au niveau des exploitations agricoles que cette question prend toute sa dimension en raison des liens avec les questions de pauvreté et de développement économique et social.

\section{La durabilité du système coton à l'échelle de l'exploitation agricole}

Les études réalisées à l'échelle des exploitations familiales permettent de comprendre les impacts économiques et sociaux de la baisse des revenus du coton depuis plusieurs années, ainsi que les stratégies des producteurs: diversification, modification des itinéraires techniques, émigration (Dufumier et Bainville, 2006). C'est à ce niveau qu'on analysera la durabilité du système coton en s'appuyant sur le modèle de durabilité des exploitations 
agricoles développé par Landais (1998) en s'attachant à prendre en considération les interrelations entre ces différents aspects : pour être durable, une exploitation agricole doit être viable (dimension économique), reproductible (dimension écologique), vivable (dimensions sociales) et transmissible (lien intergénérationnel). La remise en cause brutale de la viabilité économique des exploitations cotonnières avec la baisse des revenus des producteurs agit en interaction avec les autres composantes de la durabilité. Mais celles-ci présentaient, dès avant la crise, des signes de faiblesses, qui permettent d'expliquer l'ampleur des conséquences économiques et sociales du choc subi depuis la chute des cours du coton.

Les analyses seront faites à partir des résultats d'enquêtes auprès de petits échantillons d'exploitations agricoles. Il n'est pas question de présenter des résultats représentatifs extrapolables à l'ensemble de la région cotonnière mais bien de faire une présentation analytique du fonctionnement et des résultats des exploitations agricoles à partir d'une analyse des données collectées sur petits échantillons.

\subsection{La durabilité économique}

Le lien économique « renvoie au marché et à l'insertion de l'activité productive des exploitations dans les filières en amont et en aval, à travers les produits qu'elles mettent sur le marché » (Landais, 1998:7).

\section{La pauvreté en zone cotonnière ou «le paradoxe de Sikasso »}

Dans la zone cotonnière, cette insertion s'est faite à travers le coton en raison notamment de l'organisation de la filière qui assurait aux producteurs une garantie d'écoulement de la production à un prix connu en début de campagne et tous les services nécessaires pour la production alors que pour les autres cultures, et en particulier les céréales, les prix étaient très volatils (Samaké et al., 2007). Le développement rapide du coton laissait présumer une bonne situation économique des exploitations familiales dans cette zone, par 
rapport aux autres régions du Mali. Pourtant, la population rurale de la région de Sikasso, qui couvre une grande partie du bassin cotonnier, présente des indicateurs de pauvreté élevés, largement supérieurs à la moyenne nationale ${ }^{3}$, ce qui a conduit à parler du «paradoxe de Sikasso » (DNSI, 2007, Mesplé-Somps et al., 2008) : comment l'incidence de la pauvreté monétaire en milieu rural peut-elle atteindre $89 \%$ en 2006 dans cette région agricole, considérée comme la plus riche du pays, car excédentaire en céréales et productrice de coton?

Les revenus monétaires ont fortement diminué depuis une dizaine d'années : en monnaie courante, la marge dégagée par le coton (hors main d'œuvre et hors amortissement) est passée de 31000 FCFA par exploitation agricole et par personne en 1998/99 à 16000 FCFA en 2005/06; en 2009, elle est inférieure à celle de 1994/95 (calculs des auteurs à partir des données de la CMDT). Les résultats de l'étude RuralStruc réalisée en 2007 faisaient apparaître des marges pour le coton plus faibles que celle du maïs (Samaké et al., 2008).

Le système de culture permet, grâce à la rotation coton-céréales, d'assurer la sécurité alimentaire des populations rurales. Pourtant, un autre paradoxe est venu conforter les indicateurs de pauvreté monétaire : les enquêtes sur la sécurité alimentaire et la nutrition (PAM-UNICEF, 2005; Tefft et Kelly, 2004) ont montré une prévalence de la malnutrition chronique des enfants (rapport taille/âge) qui atteint $46 \%$ dans la zone cotonnière, c'est à dire supérieure à celle de plusieurs autres régions rurales du pays en principe plus défavorisées.

Ces indicateurs reflètent-ils une pauvreté récente due à la dégradation de la situation économique ou une pauvreté structurelle?

\section{Gains de productivité et croissance démographique}

Sur le long terme, le bassin cotonnier a été une zone attractive, qui a accueilli de nombreux migrants, notamment durant les périodes de sècheresse des années 1970 et 1980 . Sur le plan démographique, la différence entre le nord et le sud est importante. La partie nord, le «vieux bassin cotonnier » (pays Minianka) présente des densités de population élevées, 
atteignant 44 habitants/ $\mathrm{km}^{2}$ autour de Koutiala (Sangaré et al., 2006) contre moins de la moitié pour les communes autour de Bougouni, dans le sud du bassin cotonnier ; de plus, sur l'ensemble de la zone, le croît naturel reste, comme dans l'ensemble du pays, très soutenu ${ }^{4}$.

Cette augmentation de la densité de population se répercute sur la disponibilité en terres et donc sur l'évolution des exploitations. L'étude de Cissé et al. (2006) compare la productivité et les revenus des exploitations agricoles sur deux communes : l'une située dans vieux bassin cotonnier où les terroirs sont saturés et l'autre située dans une zone disposant d'importantes disponibilités foncières et où le coton est d'introduction récente. Les revenus agricoles par exploitation et par actif sont moins élevés dans le vieux bassin cotonnier que dans la zone où le coton a été introduit récemment, mais, contrairement à ce qu'on pourrait croire, ces écarts ne proviennent pas d'une différence de productivité de la terre entre les deux zones. Les rendements dans le vieux bassin sont plutôt meilleurs que sur les nouvelles terres, les exploitations ont pu capitaliser et elles sont mieux équipées, disposent d'un cheptel nettement plus important et sont mieux dotées en biens durables de consommation. Mais ces exploitations sont mises en valeur par des familles très nombreuses comptant plusieurs dizaines de membres. Pour Cissé et al. (2006) les gains de productivité ont été certes importants, mais insuffisants pour faire face à la croissance démographique, surtout à partir du moment où le terroir a été saturé. Les investissements réalisés dans d'autres activités agricoles, comme l'élevage, n'ont pas été suffisants pour compenser la stagnation de la productivité du coton. Cette situation est à l'origine des départs d'exploitations agricoles des zones saturées vers les fronts pionniers au Sud du pays (Cissé et al., 2006), mais les données manquent pour quantifier ces mouvements migratoires. Dans le contexte de croissance démographique, c'est bien la reproduction de l'exploitation qui n'est pas assurée. 


\section{Un accès au crédit de plus en plus étroit}

Au début des années 1980, les programmes d'équipement des exploitations agricoles, soutenus par la CMDT, ont permis des gains de productivité substantiels. Grâce à des prêts à faible taux d'intérêt, un grand nombre d'agriculteurs ont ainsi pu passer à la traction animale. Ce programme, réduit à la fin des années 1980, a disparu en 1987 avec le transfert de la CMDT à la BNDA (Banque Nationale de Développement Agricole) de la fonction de financement de l'agriculture. Mais, les institutions financières, BNDA ou organismes de microfinance, n'ont fait que peu de crédit d'équipement et financent plutôt la campagne agricole. Pourtant, la scission des grandes familles en petites unités de production multiplie les besoins en équipement et donc en ressources financières pour se les procurer.

Le crédit de campagne, qui permet de financer l'achat d'engrais, est actuellement lui aussi menacé. En l'absence de garantie matérielle des agriculteurs, les producteurs sont regroupés en associations qui utilisent la caution solidaire comme garantie pour obtenir des prêts des organismes financiers. Mais, avec la crise, beaucoup de producteurs ne parviennent plus à rembourser leurs crédits et de nombreuses organisations villageoises sont très lourdement endettées.

On voit donc que la durabilité économique de l'ensemble de la filière est actuellement menacée ${ }^{5}$, avec certainement des incidences sur les autres piliers du développement durable avec des pratiques pour le maintien de la fertilité des sols qui risquent d'évoluer (diminution des fertilisants) et une baisse de l'emploi rural.

\subsection{Les dimensions environnementales: causes secondaires ou conséquences de la crise du modèle?}

L'exploitation agricole est reproductible si elle permet le maintien de la fertilité du sol, de la qualité de l'eau et de l'air et le maintien d'un équilibre dans la biodiversité. L'impact des pratiques agricoles et agro-pastorales sur les ressources naturelles forme le lien écologique. 
(Landais, 1998). La durabilité écologique des systèmes de production cotonniers fait l'objet de vifs débats car les questions sont complexes. Y-a-t-il une diminution de la biodiversité et pour quelles raisons? Quel est l'impact des traitements phytosanitaires et des herbicides sur l'environnement et la santé humaine ? Comment évolue la fertilité des sols ?

\section{L'évolution des modes d'occupation de l'espace}

Les défrichements, la diminution des jachères (raccourcissement de la durée ou disparition) et les techniques de culture (notamment par le recours aux pesticides et herbicides à large spectre), ont eu des conséquences sur l'évolution de la biodiversité animale et végétale, mais peu de travaux scientifiques permettent de quantifier ces phénomènes.

L’impact des prélèvements de ligneux pour le bois-énergie est sujet de débat (Benjaminsen, 1997). Certaines études montrent l'évolution des espèces ligneuses dans deux sites du bassin cotonnier, avec, dans le vieux bassin cotonnier, une augmentation de certaines espèces qui sont considérés comme des indicateurs de sols appauvris (Cissé et al., 2006, Sangare et al., 2006). Cependant, les possibilités d'extrapolation de ces travaux très localisés restent limitées, faute d'actualisation de la carte d'occupation des sols dans la zone Sud du Mali depuis 1990.

\section{Pratiques agricoles et risques écologiques}

Le cotonnier est une plante particulièrement sensible aux ravageurs (insectes piqueurs-suceurs, champignons). L’itinéraire technique conventionnel préconisé par la CMDT prévoit de 6 à 8 traitements de pesticides par cycle cultural. La contamination des sols, de l'eau et des organismes vivants par les produits phytosanitaires est donc un problème sérieux au moment de l'épandage, mais aussi par les résidus qu'il laisse. L'étude de Dem et al. (2007) montre que les échantillons d'eau et de sol étudiés contiennent de résidus de pesticides, notamment d'endosulfan (substance active de pesticide très largement utilisé en zone 
cotonnière, mais interdit depuis quelques années); sans surprise, les concentrations les plus élevées se trouvent dans le vieux bassin cotonnier (Koutiala). Les résidus sont actuellement sous le seuil limite admis; cependant, beaucoup de relevés effectués à proximité des habitations et des zones de stabulation des animaux montrent une contamination, ce qui pose des problèmes sanitaires ${ }^{6}$. Les auteurs soulignent aussi le manque de documentation disponible à l'échelle du bassin cotonnier. Malgré plusieurs rapports soulignant la toxicité des produits utilisés (PAN 2004, Ton 2006), l'impact sur la santé des populations et l'environnement de l'utilisation des produits phytosanitaires en zone cotonnière n'a pas, jusqu'à ce jour, mobilisé de nombreuses recherches, contrairement à ce qu'on observe dans les pays développés.

\section{Le maintien de la fertilité des sols hypothéqué ?}

La question de la fertilité des sols est assez bien documentée, en raison certainement de l'intérêt économique direct lié à la baisse des rendements observée depuis le mieux des années 1990 (figure 2) dont on a recherché les causes dans un éventuel appauvrissement des sols. Selon certains auteurs, le passage à une agriculture permanente, contraint par l'augmentation de la densité de population et l'expansion des superficies cultivées notamment en coton, entraînerait l'épuisement minéral des terres (Van Der Pol, 1992). Pour d'autres, la culture permanente est liée, avant tout, aux changements des techniques culturales ; Gigou et al. (2004) observent que dans des zones où il existe encore de fortes disponibilités foncières, qui permettraient les jachères de longue durée, les producteurs optent pour la culture permanente au moins pour une partie de leur surface cultivée. La culture attelée et les pratiques de fertilisation le leur permettent; ils valorisent ainsi l'important investissement que représentent le défrichement et le dessouchage. Selon ces auteurs, les champs les plus anciennement cultivés sont loin d'être épuisés et présentent même de meilleurs rendements que ceux nouvellement défrichés : cela peut s'expliquer par un bon 
amendement, mais aussi par le choix des sites, les meilleures terres sont mises en valeur les premières.

Le maintien, voire l'amélioration de la fertilité des sols sont des processus dynamiques qui dépendent des pratiques paysannes en liaison avec des facteurs autant techniques (recherche agro-pédologique, définition d'itinéraires techniques) qu'économiques (approvisionnement en intrants minéraux, arbitrage des coûts de production par les producteurs) ou socio-économiques (intégration agriculture-élevage). C'est dans ce contexte que doit être replacée la baisse régulière des rendements du coton depuis le début des années 1990 : d'après les données de la CMDT, on est passé en 15 ans d'un rendement moyen de $1200 \mathrm{~kg}$ de coton-graine /ha à $900 \mathrm{~kg} / \mathrm{ha}$ (figure 2).

Les causes de cette baisse sont multiples (Fok, 2007). Parmi celle-ci, la mise en valeur, dans les terroirs les plus peuplés, de terres de moins bonne qualité. Mais, d'autres facteurs jouent aussi. En premier lieu, les producteurs surévaluent les surfaces car cela leur donne accès à une quantité plus importante d'intrants minéraux. Ensuite, une partie de ces engrais peut être utilisée sur les cultures vivrières au détriment du coton. Le renchérissement du coût des intrants minéraux et la baisse des prix du coton, se traduit par une forme de « désintensification » de la culture du coton.

A côté des engrais minéraux, la fumure organique provenant de l'élevage et du compostage est tout aussi essentielle pour l'amendement des champs. Elle nécessite de la disponibilité en bétail mais aussi des moyens pour garder le bétail sur place (pâturages et/ou fourrages, techniques de stabulation), ce qui devient de plus en plus difficile en raison de la diminution des terrains de parcours, ainsi qu'une maîtrise technique de la valorisation de la fumure organique. 
Les insuffisances dans les restitutions d'éléments minéraux et organiques compromettent le maintien de la fertilité des sols. Cette question de la fertilité des sols se trouve donc au centre des interrelations économiques et environnementales.

Si les faiblesses du système cotonnier en matière de durabilité environnementale ne sont pas les principales causes de la crise actuelle, elles n'en constituent pas moins des facteurs aggravants, qui risquent de se trouver amplifiés dans les évolutions institutionnelles récentes, comme la diminution de l'appui en formation des producteurs.

\subsection{Le lien social : une construction inachevée ?}

La qualité du lien social est une autre condition de la durabilité : elle permet que l'environnement social soit « vivable ». Cela renvoie à l'insertion des producteurs et de leurs familles «dans les réseaux principalement locaux de relations non marchandes, relations avec les autres agriculteurs comme avec l'ensemble des acteurs sociaux » (Landais, 1998).

\section{L'inégalité infructueuse?}

Selon de nombreux auteurs, la différenciation sociale entre producteurs s'est accrue : créateur de richesse, le «système-coton » a aussi maintenu, voire creusé les inégalités intravillageoises, au moins durant la phase d'expansion de la culture cotonnière dans des terroirs non saturés (Dufumier et Bainville 2006; Devèze et Halley des Fontaines 2005). Les exploitations les mieux dotées en facteurs de production (foncier, cheptel, main d'œuvre) ont le plus profité du système coton et fait progresser leurs revenus monétaires (Wodon et al., 2006). Lorsqu'elles disposaient de droits fonciers traditionnels, elles ont pu s'étendre. Cependant le modèle de développement du coton a été très inclusif, laissant peu de monde au bord de la route, le taux élevé d'équipement en traction animale des exploitations dans le vieux bassin est ici un bon indicateur (Samaké et al., 2008). 
Mais, la pression foncière accrue dans le vieux bassin a eu pour conséquence une réduction des ressources dans les espaces sous gestion communautaire. Ainsi, on observe une réduction des espaces accessibles à tous les villageois pour faire pâturer le bétail, alors que le nombre d'animaux à nettement progressé. Cela est d'autant plus sensible que les grands troupeaux sont concentrés entre les mains d'une minorité qui accapare ainsi la ressource pastorale. La saturation des terroirs dans certaines zones du bassin cotonnier accroît les tensions entre les familles issues de lignages plus anciennement installés, ayant une forte préhension sur le foncier, et d'autres familles arrivées plus récemment.

Dans les exploitations de grande taille démographique où cohabitent plusieurs générations, les champs individuels laissés aux femmes ou aux jeunes hommes ont diminué au profit des champs collectifs gérés par le chef d'exploitation. Lorsque la pression foncière s'accroît, les droits d'usage les plus précaires sont les premiers à disparaître et ce sont les champs des femmes et des jeunes qui sont les plus concernés et les tensions que la situation entraîne conduisent quelquefois les jeunes ménages à quitter la concession (Devèze et Halley des Fontaines, 2005).

\section{Les organisations de producteurs, des géants aux pieds d'argile}

Les organisations de producteurs de coton d'Afrique de l'Ouest sont souvent citées comme exemple de capacité de structuration du milieu rural et d'émergence d'un réel pouvoir de négociation émanant du milieu paysan. Elles sont devenues des acteurs essentiels dans le paysage institutionnel du pays.

Cependant, au Mali, les associations villageoises initiées par la CMDT ont longtemps été limitées à une délégation de fonctions techniques et à une prise en charge collective de certains risques, comme dans le cas du crédit à caution solidaire. Les associations se sont trouvées fragilisées par de nombreux problèmes de gestion financière : des revenus collectifs 
ont fréquemment été consacrés au remboursement des crédits individuels de quelques membres défaillants, ce qui ne manquait pas aussi d'avoir un coût social (Jonckers, 1994).

La transformation de toutes les associations villageoises en coopératives de producteurs de coton (APC) a été faite dans la perspective de la représentation des producteurs au sein de la filière (il est prévu qu'ils disposent d'une part des actions de manière à être présents dans les conseils d'administration des sociétés privées régionales). Aujourd'hui la réduction des superficies en coton et du nombre d'exploitations agricoles «cotonnières », les difficultés rencontrées pour faire jouer la caution solidaire et la spécialisation cotonnière remettent en question la capacité et la légitimité de ces organisations vis-à-vis du développement local.

Le lien social a été très fragilisé et la durabilité sociale menacée à plusieurs échelles : à l'intérieur d'une même exploitation vis-à-vis des cadets sociaux (femmes, jeunes), entre exploitations plus ou moins aisées et équipées, au sein des associations de producteurs et entre celles-ci et la CMDT en attendant les sociétés privées régionales appelées à la remplacer.

\subsection{La transmissibilité en question}

Une des dimensions fondamentales de l'agriculture familiale est celle de la transmission d'une exploitation d'une génération à l'autre. Cette transmissibilité « représente un idéal de solidarité entre les générations, qui est au cœur du développement durable »(Landais, 1998). Au Mali, le maintien à un degré assez élevé de cette capacité de transmission et de pérennisation des agricultures familiales est essentiel : les activités en milieu rural restent très centrées sur l'agriculture et l'élevage, l'offre d'emplois urbains stagne très en deçà de la demande, et les possibilités d'émigration sont réduites que cela soit dans la sous-région (par exemple avec le conflit en Côte d'Ivoire) ou hors du continent africain.

On observe «un recul de l'autorité traditionnelle qui se manifeste par une réduction du temps consacré aux travaux collectifs, un éclatement des familles lié à une évolution des valeurs sociétales, et en particulier les cadets qui s'opposent de plus en plus aux aînés» 
(Cissé et al., 2006). L'éclatement en petites exploitations pose des problèmes dans le contexte actuel de difficulté d'investissement : leur équipement est insuffisant et les accès aux crédits de plus en plus difficiles, par conséquent, leurs résultats technico-économiques sont médiocres. Plusieurs études montrent la part importante des exploitations familiales en situation d'insécurité alimentaire (PAM/UNICEF, 2005, ODHD/PNUD, 2007, Sidibe et al., 2007). La viabilité économique est donc encore plus difficilement accessible et la transmissibilité fortement compromise.

Landais (1998) note aussi l'importance des représentations que la génération de futurs exploitants agricoles peut avoir du métier. Actuellement, dans le bassin cotonnier, cette image se trouve fortement dégradée, et pour une grande partie de la jeune génération, le coton est associé à la pauvreté, contrairement à l'image de réussite qu'il pouvait véhiculer il y a deux décennies.

\section{Effondrement ou rebond du système cotonnier?}

\subsection{Une embellie des prix mondiaux}

Après une déprime prolongée des prix, de la fin des années 90 jusqu'à la crise des prix des denrées alimentaires en 2008 , le cours du coton-fibre est fortement remonté sur le marché mondial, doublant entre mars 2009 et octobre 2010 et poursuivant son ascension rapide en début 2011 (506 cents US\$/kg en mars 2011). Cette inversion de tendance a plusieurs causes : des mauvaises récoltes en 2009/10 liées à des aléas climatiques dans quelques grands pays producteurs, comme la Chine, le Pakistan et le Brésil, une diminution des stocks mondiaux et une hausse de la demande intérieure en Chine et dans les pays émergents. Cette dernière raison, plus structurelle, conduit les analystes à estimer que les cours garderont un niveau relativement élevé dans les années à venir.

Cependant, les producteurs de la zone CFA restent liés à l'évolution du rapport entre l'euro, monnaie à laquelle le FCFA est arrimée, et le dollar US, monnaie dans laquelle sont 
fixés les cours mondiaux. La figure 3 indique les évolutions comparées du prix international moyen de coton fibre exprimé en US \$ et en FCFA en prenant comme base 100 l'année 1994 (année de la dévaluation du CFA par rapport à l'euro). Si la faiblesse de l'euro par rapport au dollar a limité la baisse durant les années 1999 à 2003, le taux de change euro/dollar, n'a pas été favorable au coton malien pendant les années qui ont suivies.

La baisse des prix au producteur a atteint un prix-plancher de $160 \mathrm{CFA} / \mathrm{kg}$ en 2006 , entraînant avec elle une chute de la production de coton-graine. En amélioration depuis 2009, le prix d'achat est annoncé pour la campagne 2011/2012 à 255 CFA/kg pour la catégorie supérieure, soit une augmentation de 70 CFA par rapport à la campagne précédente. L'amélioration est donc très significative sur les prix, mais avec une part non négligeable de facteurs conjoncturels. Jusqu'ici, la production peine à reprendre ce qui montre que les acteurs de la filière ne peuvent absorber aussi rapidement de telles réorientations. Cette adaptation est rendue d'autant plus laborieuse que la restructuration de la filière, engagée en 2001, n'est pas encore achevée dix ans plus tard.

\subsection{Une longue restructuration de la filière}

La restructuration de la filière a démarré après la «grève des semis » de coton en 2000. Tirant les leçons des libéralisations engagées dans d'autres pays, cette restructuration a été organisée en plusieurs étapes. Elle a commencée par un désengagement de la CMDT des opérations qui n'étaient pas directement liées à la production cotonnière : entretien des pistes rurales, appui aux services de santé et d'éducation, formation technique des producteurs. L'approvisionnement en intrants dits non stratégiques (notamment les engrais pour les céréales) a été transféré aux organisations de producteurs. Cette décision peut être interprétée comme la volonté d'affirmer la voie choisie vers la privatisation. Elle a été mise en œuvre avec beaucoup d'impréparation; le transfert s'est fait dans un premier temps vers les syndicats de producteurs regroupés, seules organisations, au début des années 2000, pouvant 
revendiquer la représentation des producteurs de coton et de leurs organisations de base. Par la suite, des actions ont été menées pour transformer les organisations de base en coopératives et s'assurer de leur fédération en une organisation susceptible de représenter les producteurs de coton dans toutes les institutions prévues pour une gestion «interprofessionnelle de la filière ».

La phase de privatisation de la CMDT a été plusieurs fois reportée. Cette dernière opération est contestée par une partie des acteurs politiques et économiques maliens : leurs réticences, voire leur opposition, sont motivées par les déboires d'autres privatisations d'entreprises publiques au Mali (notamment Huicoma ou les Chemins de Fer). Les performances économiques de la CMDT, monopole public, sont souvent jugées faibles en comparaison avec d'autres opérateurs (Tschirley 2009 et 2010). La chute des prix sur le marché mondial, survenue en plein processus de privatisation de la CMDT, a fortement grevée la situation financière déjà très dégradée de l'entreprise, la rendant peu attractive. Pour opérationnaliser la cession d'une entreprise de cette taille, la CMDT a été divisée en 4 filiales (Holding CMDT) correspondant à différentes zones agro-écologiques, dotées chacune d'usines d'égrenage. Le capital de chaque filiale doit être cédé à hauteur de $61 \%$ à un opérateur privé par appel d'offres en 2011 ; malgré la hausse importante des prix du coton sur le marché mondial en 2011 et l'apurement des dettes de la CMDT, les candidats à la reprise restent prudents.

\subsection{Un avenir incertain}

La remontée des cours mondiaux du coton permet aux dirigeants de la CMDT de tenir un discours mobilisateur et optimiste sur l'avenir de la filière. Pour inciter les producteurs à revenir vers le coton, des mesures attractives sont prises : subvention des engrais, réforme du mécanisme de financement avec la suppression de la caution solidaire pour le crédit de campagne, hausse des prix d'achat au producteur. Les délais de paiement qui constituent une 
des raisons de désaffection des producteurs pour la culture de coton, doivent être raccourcis. Cependant, le rebond attendu n'est pas encore perceptible à l'échelle des champs : en 2011, les surfaces semées en coton et les volumes produits sont encore à environ la moitié des niveaux atteints au milieu des années 2000.

Le système coton est-il en mesure de retrouver sa dynamique des années 1990 ? Plusieurs questions conduisent à tempérer l'optimisme qui perle dans certains discours prédisant le retour de "l'or blanc". En effet, les piliers du système sont atteints. D'abord les infrastructures physiques : l'appareil industriel est dégradé par un manque d'entretien durant ces dernières années et les pistes rurales ne sont plus - ou mal - entretenues. Ensuite, les activités de formation et d'encadrement des producteurs ont quasiment disparu, entraînant une perte de savoir-faire technique, alors même que le Mali pourrait se tailler une niche en misant sur la qualité du coton. Les rapports sociaux sont détériorés et la confiance a disparu à différents échelons : entre producteurs et société d'encadrement, entre villageois eux-mêmes suite aux problèmes d'endettement mutualisés par la caution solidaire.

L'incertitude quant au devenir de l'organisation tant en amont qu'en aval de la production entretient un sentiment d'insécurité chez les producteurs qui ont dû s'adapter pour faire face à la crise cotonnière : changement d'assolement et de pratiques agricoles, réorganisation pour l'approvisionnement, recherche d'autres sources de revenu monétaire ; il n'est donc pas évident qu'ils répondent massivement et rapidement aux incitations que représente le prix. Pour certains, ce sera d'autant plus difficile qu'il y a parfois eu une décapitalisation au niveau de l'exploitation ne permettant pas de reprendre la culture de coton : le matériel agricole et les bœufs pour la culture attelée ont été vendus pour rembourser les dettes et des jeunes actifs sont partis en migration.

Dans ce contexte, l'arrivée de sociétés privées exacerbe les craintes. Le désengagement de l'Etat, dans cette configuration de «libéralisation partielle » (Kydd et al., 
2004), laisse donc craindre une amplification des risques de coordination où les acteurs du marché, jouant à très court terme, ne respectent pas les règles minimales qui assurent la pérennité même de la filière cotonnière.

\section{Conclusion}

Depuis trois décennies, l'expansion de la production cotonnière a profondément transformé les paysages, le système agraire ainsi que l'organisation économique et sociale de toute la partie sud du Mali, celle qu'on appelle le bassin cotonnier. Les acquis en termes de développement sont importants.

Examiné sous l'angle de la durabilité, ce système présentait des atouts, mais aussi des faiblesses, aussi bien sur les plans environnementaux qu'économiques et sociaux. Ainsi, avant même la chute des prix mondiaux du coton, 1e système de production basé sur cette culture de rente qualifiée il y a peu encore d' «or blanc » présentait des signes de vulnérabilité suscitant des interrogations sur sa durabilité dans les écosystèmes de savane en raison notamment d'une stagnation, voire d'une diminution, de la productivité.

Avec la baisse des cours mondiaux la durabilité générale du système a été largement remise en question. Aujourd'hui, le contexte dans lequel les producteurs doivent développer des stratégies d'adaptation ou d'innovation est marqué par l'incertitude : incertitudes sur les prix du coton, incertitudes sur l'évolution du cadre institutionnel avec la privatisation de la société cotonnière en cours et les questions qu'elle soulève sur la réorganisation du crédit et du conseil par exemple, risques sur les autres filières de production pour lesquels les appuis techniques et organisationnels sont encore limités et concentrés sur quelques zones ${ }^{7}$. Par ailleurs, la situation de pauvreté en capital financier, physique et humain limite fortement les possibilités d'investissement et de diversification durable. Beaucoup de producteurs sont précipités dans des stratégies de survie non durables sur le plan environnemental comme le prélèvement accru sur les ressources. 
L'enjeu actuel est donc la restauration de la compétitivité de la filière coton tout en développant la productivité globale des exploitations agricoles, en diversifiant leurs sources de revenu, et en veillant au maintien d'un équilibre social et environnemental. L'autre enjeu, qui contribue à la durabilité du système, est d'assurer ou de restaurer dans le cadre de la décentralisation les missions de services publics (santé, éducation, infrastructures routières, formation agricole) qui étaient jusqu'ici en partie prises en charge par le «système coton », c'est-à-dire soit directement par la CMDT, soit par les associations villageoises. Sans une réelle politique publique, la récente hausse des prix aux producteurs ne sera pas suffisante pour enrayer efficacement la dégradation de la situation économique, ce qui pourrait conduire à une catastrophe sociale et environnementale.

\footnotetext{
${ }^{1}$ Le classement de l'IDH (indicateur de développement humain) du Mali établi par le PNUD en 2010 place le pays au 160ème rang sur 169 pays.

${ }^{2}$ On notera qu'en raison de la baisse de la pluviométrie dans les années 1970, la culture du coton a été abandonnée dans une frange au Nord-Est de la région actuelle.
}

${ }^{3}$ Une étude de l'INSTAT de 2011 compare les principaux indicateurs de pauvreté calculés à partir des enquêtes nationales réalisées en 2001, 2006 et 2011. Cette étude conclut à une diminution de l'incidence de la pauvreté entre 2001 et 2010, pour l'ensemble du pays, à l'exception de la région rurale de Sikasso. L'incidence de la pauvreté était, d'après l'INSTAT, de $56 \%$ en $2001,57 \%$ en 2006 et $44 \%$ en 2010 (milieu rural et milieu urbain) alors que pour ces trois enquêtes, l'incidence de pauvreté dans la population rurale de la région de Sikasso dépasse toujours $80 \%$ (INSTAT, 2011).

${ }^{4}$ D'après le RGPH (recensement général de la population et de l'habitat) de 2009, la population de la région de Sikasso a été multipliée par près de 1,5 depuis 1998 , ce qui représente un taux de croissance annuel moyen de 3,6\%. Le taux de fécondité est de 7,3 enfants par femme au Mali (INSTAT, 2009).

${ }^{5}$ En 2009 et 2010, les intrants ont été subventionnés dans la foulée de l'initiative Riz de 2008 (mesures prises pour stimuler la production agricole et faire face à la crise alimentaire). Mais, si cette mesure bénéficie effectivement aux producteurs, il est toutefois peu probable que ce système soit viable à moyen et long terme.

${ }^{6}$ Cette étude récente a été réalisée sur 60 échantillons de sol.

${ }^{7}$ Certains partenaires techniques et financiers, comme la coopération suisse, appuient la diversification des filières agricoles dans la région de Sikasso (mangue etc.) et la diversification de la production cotonnière sur un marché de niche comme le coton biologique et équitable. L'AFD appuie la production de coton équitable dans la région de Kita. 


\section{Bibliographie}

Bélières, J-F., Benoit-Cattin, M., Barret, L., Djouara, H., et Kébé, D. (2008) Les organisations de producteurs en zone cotonnière au Mali. Conditions d'émergence et perspectives. Economie Rurale, 303-304-305 : 22-38.

Belloncle, G. (1993) Anthropologie appliquée et développement associatif. Trente années d'expérimentation sociale en Afrique sahélienne (1960 - 1990). Paris, L'Harmattan, Anthropologie appliquée, $184 \mathrm{p}$.

Benjaminsen T. (1997) Is there a fuelwood crisis in rural Mali ? GeoJournal 43 : 163-174.

Cissé, I., Maïga, A.Y., Bélières, J.F., Traoré, A. et Kounkantji, B. (2006) Croissance démographique, développement de la culture du coton et gestion durables des ressources naturelles en zone Mali Sud. Programme PRIPODE Institut d'Economie Rurale du Mali, $118 \mathrm{p}$.

CPS (2008) Recensement général de l'agriculture campagne agricole 2004/05. Résultats définitifs.. Cellule de planification et de statistique du Ministère de l'agriculture. Bamako, $139 \mathrm{p}$.

Dem, S.B., Cobb, J. and Mullins, D. (2007) Pesticide Residues in Soil and Water from Four Cotton Growing Areas of Mali, West Africa. Journal of Agricultural, Food and Environmental Sciences, 1(1) : 1-16.

Deveze, J-C. etHalley des Fontaines, D. (2005) Le devenir des agricultures familiales des zones cotonnières africaines : une mutation à conduire avec tous les acteurs. Document AFD, Paris, 42 p.

Djouara, H., Bélières, J-F. et Kébé, D. (2006) Les exploitations agricoles familiales de la zone cotonnière du Mali face à la baisse des prix du coton graine. Cahiers Agricultures 15 (1) : $64-71$.

DNSI (2007) Tendances et déterminants de la pauvreté au Mali(2001-2006), Bamako, 38 p.

Dufumier,M. et Bainville, S. (2006) Le développement agricole du Sud-Mali face au désengagement de l'Etat.Afrique contemporaine 217 : 121-133. 
Egg, J., Gabas, J-J., Bélières, J-F., Bosc, P-M., Cissé I., Diallo, B., Mainguy, C., Traoré, B. (2008) Trajectoires de construction des politiques agricoles au Mali. Document de travail du projet Propocid, 4/08, 61 p.

Fok, M. (1994) L'évolution du système coton au Mali. Cahiers Agricultures 3 (5) : 329 - 336.

Fok, M. (1999)Histoire du développement de la filière cotonnière au Mali. Rôle et place des innovations institutionnelles.Rôle et place de la recherche pour le développement des filières cotonnières en évolution en Afrique, CIRAD, Montpellier, 1-2 Septembre 1999, pp. $19-26$.

Fok, M. (2007) Analyse multifactorielle de la baisse des rendements du coton en zone CMDT : de la physiologie à l'idéologie. CIRAD, 158 p.

Gigou, J., Giraudy, F., Doucouré, C.O., Healy, S., Traoré, K., Guindo, O.(2004) L’âge des champs : un indicateur du passage de la culture itinérante à la culture permanente dans le bassin cotonnier du Mali. Cahiers de l'Agriculture (13) : 467-472.

Hugon, P. (2005a). Les filières cotonnières africaines au regard des enjeux nationaux et internationaux. Notes et Études Économiques (23) : 87-112.

Hugon, P. (2005b) Les réformes de la filière coton au Mali et les négociations internationales. Afrique contemporaine (216) : 203-224.

INSTAT (2009) 4ème Recensement Général de la Population et de l'Habitat du Mali, Fiche de synthèse.R.G.P.H. 2009, Bureau central du recensement, 4 p.

INSTAT (2011) Enquête par grappe à indicateurs multiples et de dépenses des ménages MICS/ELIM 2010. Résultats préliminaires du volet ELIM, Bamako, 114 p.

Jonckers, D. (1994)Le mythe d'une tradition communautaire villageoise dans la région Mali Sud. In : Jacob, J.-P. et Lavigne Delville, P. (eds) Les associations paysannes en Afrique. Paris, APAD - Karthala - IUED, pp.121 - 134.

Kébé, D. et Sidibé-Kébé, M-C. (1998) Etude diagnostic de la crise des associations villageoises en zone CMDT. Rapport de recherche. IER/ESPGRN-Sikasso. Bamako, 60 p. 
Kydd, J., and Dorward, A. (2004) Implications of market and coordination failures for rural developments in least developed countries. Journal of international Development. (16) : 951-970.

Landais, E. (1998) Agriculture durable: les fondements d'un nouveau contrat social ?Le courrier de l'environnement (33) : 5-22.

Mathieu, P. (2001) Accroissement démographique et gestion de l'environnement en Afrique sub-saharienne. Savoirs et jeux d'acteurs pour des développements durables. Louvain la Neuve, Population et Développement (9) :375-411.

MDR (2001) Lettre de politique de développement de la filière coton. République du Mali Ministère du développement rural. Bamako, 19 p.

Mesplé-Somps, S., Robilliard, A-S, Gräb, J., Cogneau, D. et Grimm, M. (2008) Coton et pauvreté en Afrique de l'Ouest : analyse comparée des conditions de vie des ménages au Mali et au Burkina Faso. AFD, Département de la Recherche, Série Analyse d'Impacts, (1) $82 \mathrm{p}$.

Nubukpo, K. (2011) L'économie politique de la réforme des filières cotonnières d'Afrique de l'Ouest et du centre : vers la convergence des modes d'organisation ? Mondes en développement, 3 (155) : 93-109.

ODHD/PNUD (2007) Analyse de la pauvreté alimentaire au Mali. Observatoire du Développement Humain Durable et de la Lutte Contre la Pauvreté au Mali / Programme des Nations Unies pour le Développement. Bamako, 92 p.

ODHD (2009) Contribution du coton à la croissance économique du Mali, Bamako, 91 p.

OECD/Sahel and West Africa Club (2006) The Development Dimension - Cotton in West Africa: The Economic and Social Stake. Paris, OECD,127 p.

PAM-UNICEF (2005) Analyse de la sécurité alimentaire et de la vulnérabilité (CSFVA), Rapport CSFVA. Bamako, 94 p.

PAN (2004) Pesticid Action Network Africa. Rapport annuel 2004, 25 p. 
Raymond, G. (1989) L'importance du coton dans les systèmes de production en zone de savane d'Afrique de l'Ouest et du Centre. In : Economie des Filières en Régions Chaudes. Formation des prix et échanges agricoles. CIRAD, Montpellier, 11-15 septembre 1989, pp.195-210.

Samaké, A., Bélières, J-F., Bosc, P-M., et Sanogo, O. (2007) Les implications structurelles de la libéralisation sur l'agriculture et le développement rural au Mali. Première phase du programme RuralStruc - Synthèse nationale- MALI. CEPIA / Banque Mondiale \& Coopération française. Bamako. 278 p.

Samaké, A., Bélières, J-F., Corniaux, Ch., Dembélé, N., Kelly, V. Marzin, J., Sanogo, O. et Staatz J., 2008. Dimensions structurelles de la libéralisation pour l'agriculture et le développement rural. Phase II du programme RuralStruc MALI IER/CIRAD/MSUBanque Mondiale/Coopération française/FIDA. Bamako. 363 p.

Sangare, M. , Poccard-Chapuis, R., Blanchard,M., Bengaly, M., Koukandji, B., Djouarra, H ., Coulibaly, N., Senou, O. et Coulibaly, D. (2006) Situation et dynamiques agropastorale de Dentiola : Diversité et pratiques. Projet DCG2-50 de Duras, CIDES-IER., 52 p.

Sidibe, M., Belières, J-F. etCoulibaly, J. (2007) Gestion des stocks et de la trésorerie dans les exploitations du vieux bassin cotonnier : pratiques et contraintes. Rapport PCP-GESED, IER, IPR-IFRA, CIRAD.

Tefft, J. andKelly, V. (2004)Understanding and Reducing Child Malnutrition in Mali : Interim Research Findings for the Project on Linkages between Child Nutrition and Agricultural Growth (LCNAG).Department of Agricultural Economics, Michigan State University, Staff paper 2004-27, $70 \mathrm{p}$.

Ton, P. (2006) Promouvoir la production plus durable de coton : Possibilités au Burkina Faso et au Mali. Rapport UNEP-FAO, 70 p.

Tschirley, D.L., Poulton, C. and Labaste, P. (eds) (2009) Organization and performance of cotton sectors in Africa: learning from reform experience. Washington DC, World Bank, $233 \mathrm{p}$. 
Tschirley, D.L., Poulton, C., Gergey,, N., Labaste, P., Baffes, J., Boughton, D. et Estur, G. (2010) Institutional Diversity and Performance in African Cotton Sectors. Development Policy Review, 28 (3) : 295-323.

Van Der Pol,F. (1992) Soil mining. An unseen contributor to farm income in southern Mali. Amsterdam, Royal Tropical Institute, Bulletin 325.

Wodon, Q., Briand, V., Labaste, P., Nouve, K. et Sangho, Y. (2006) Cotton and poverty in Mali. Banque Mondiale, Working paper, Washington, 161 p.

Figure 1 : Localisation de la zone cotonnière au Mali

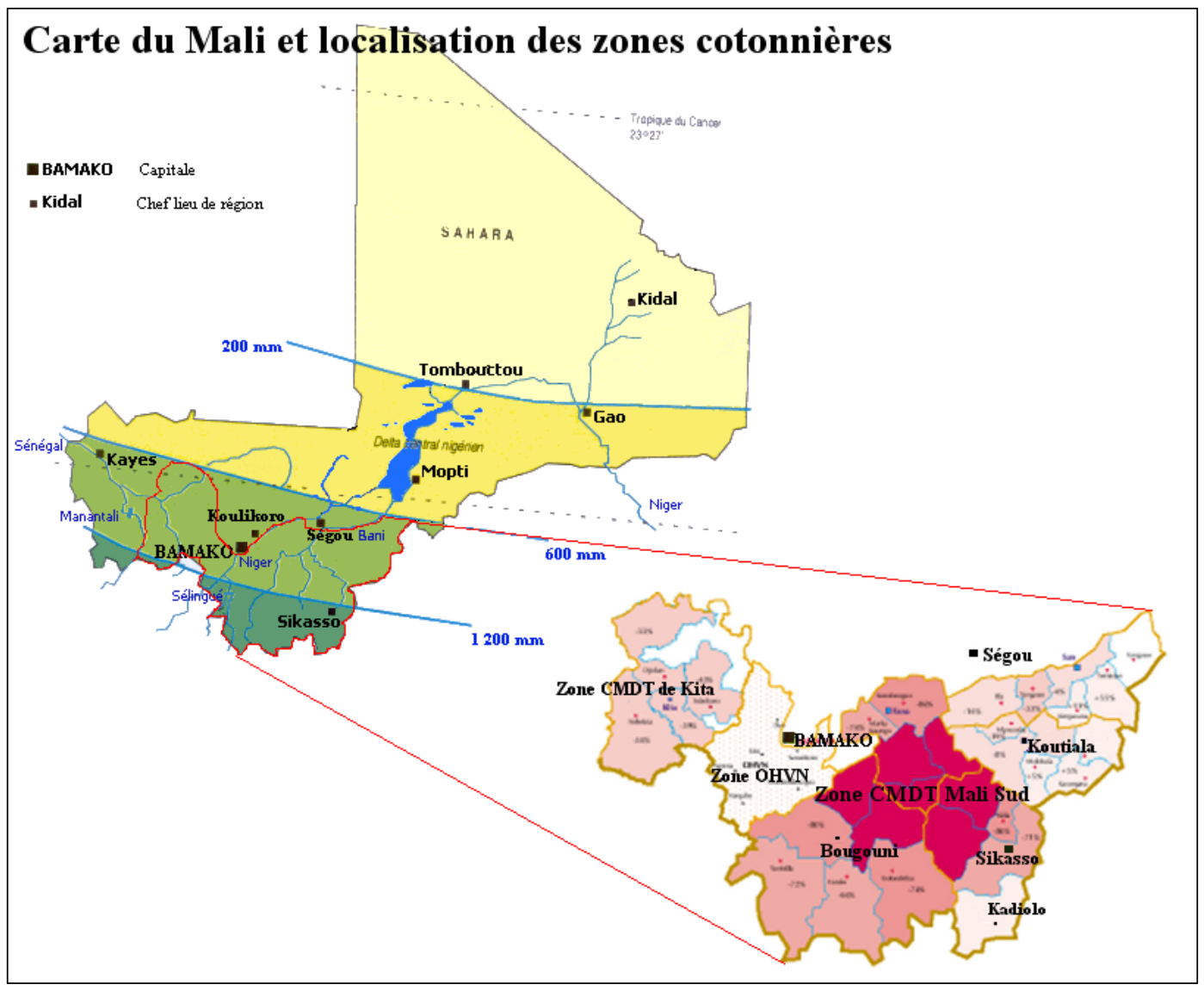

Source : Djouara, H., Bélières, J-F. et Kébé, D. (2006) 
Figure 2 : Evolutions des superficies cultivées en cotonnier, de la production et des rendements en coton graine de 1960 à 2009

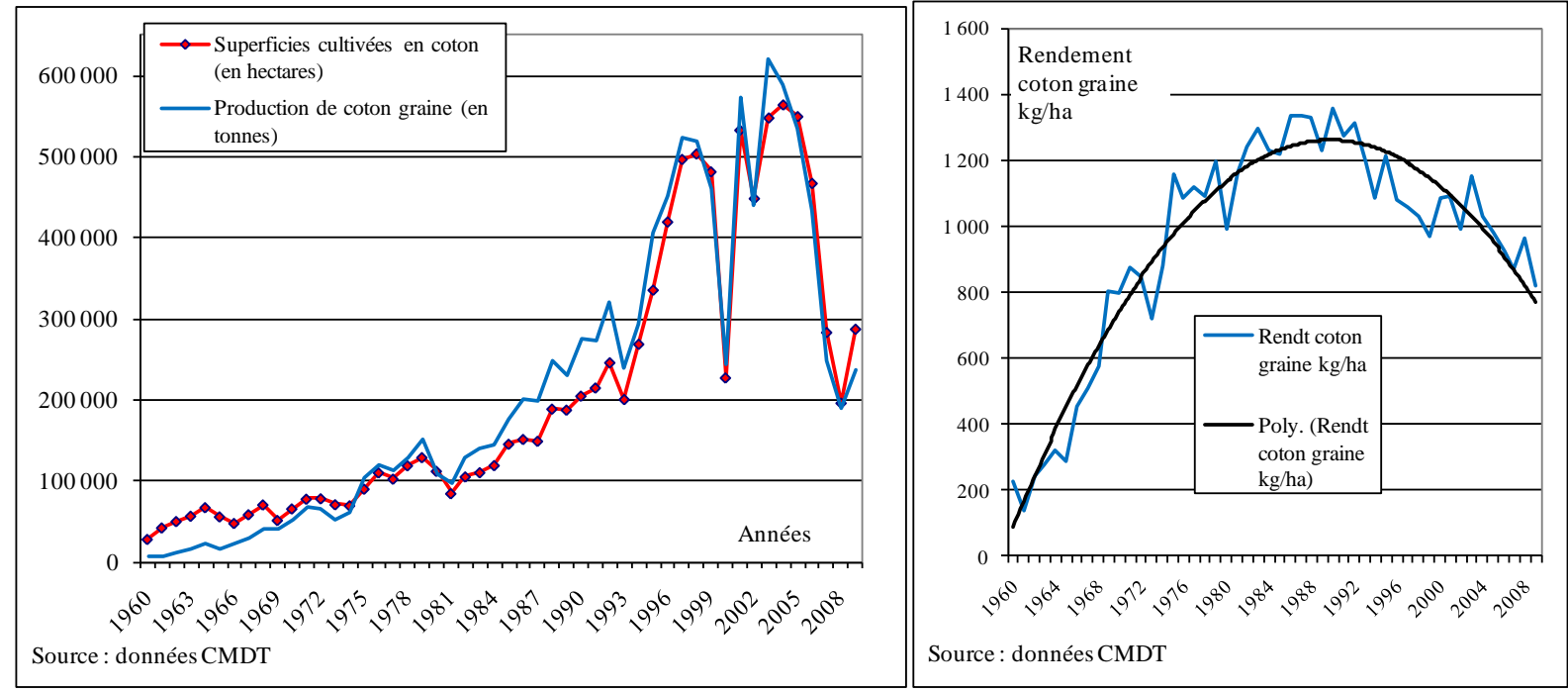

Figure 3 : Evolution du prix moyen annuel de la fibre de coton sur le marché international en US \$ et en CFA (1990/2010, base 100 en 1994)

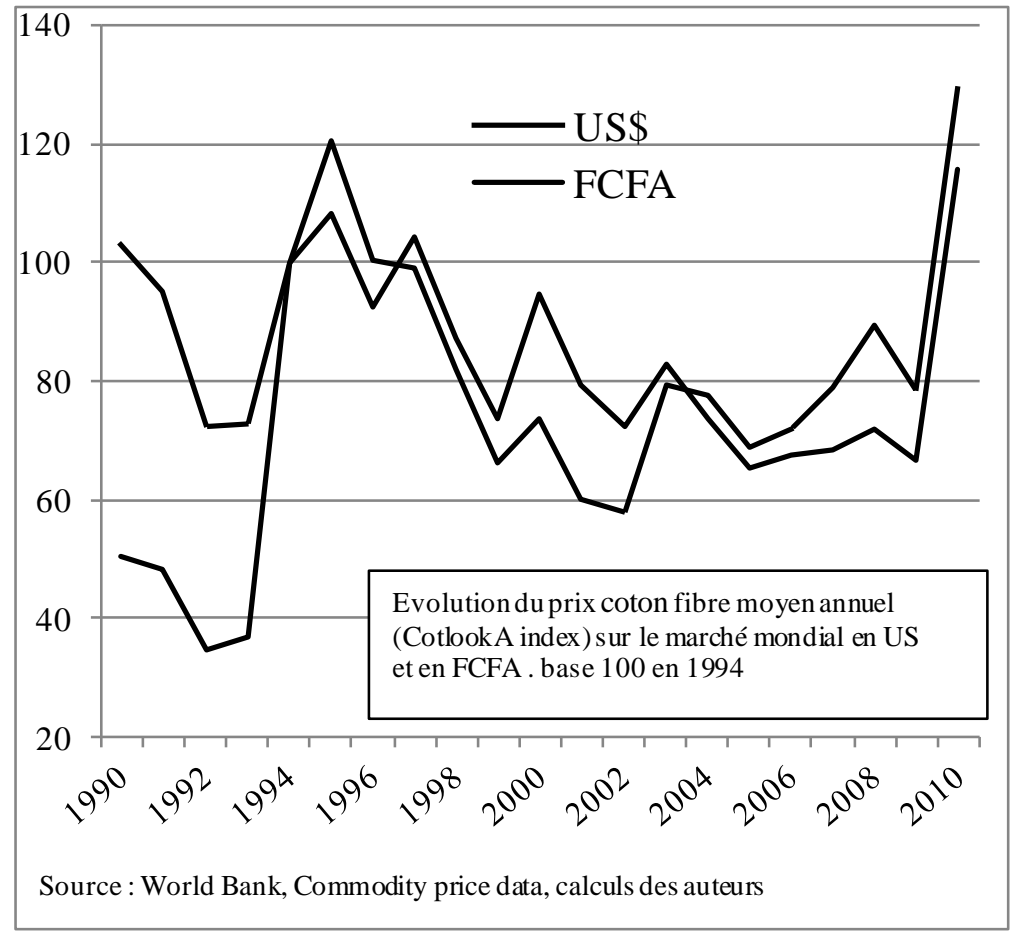


Figure 4 : Evolution de la production du coton graine et du prix payé au producteur au Mali (1994-2009)

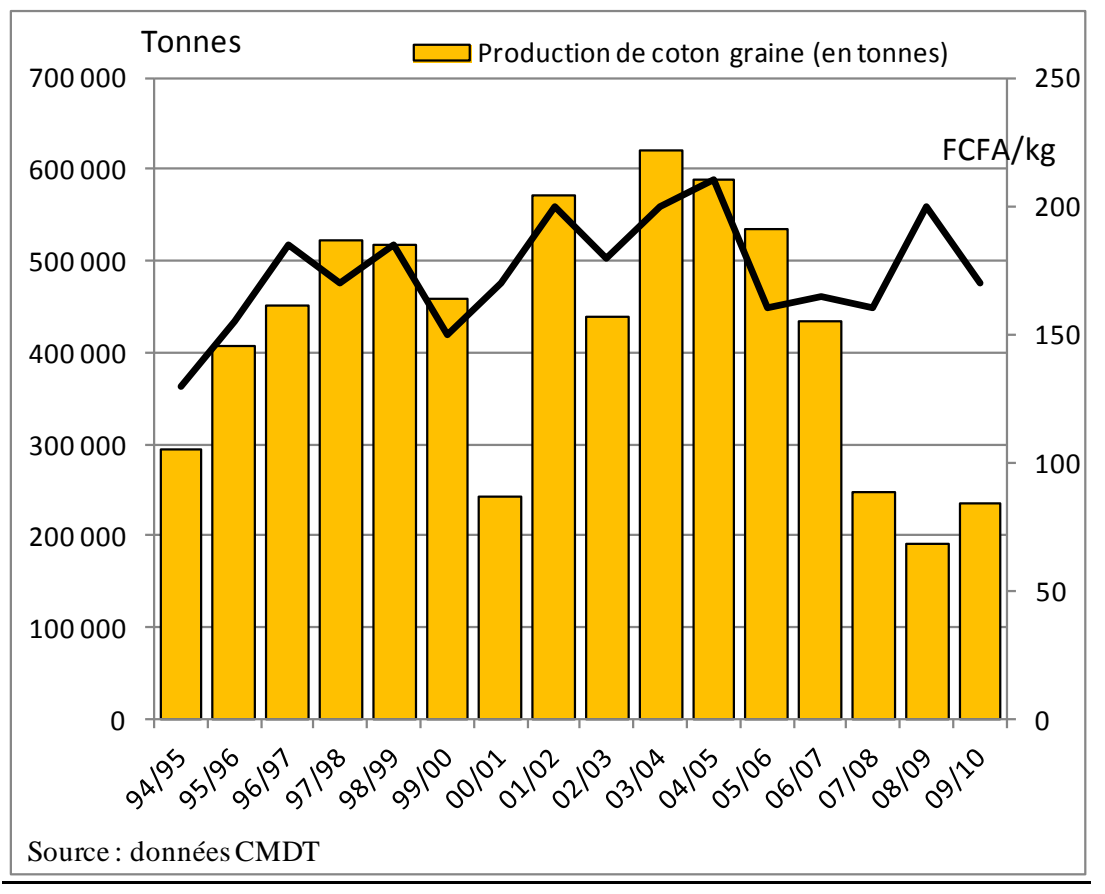

